Artículo de investigación científica y tecnología.

Cómo citar: J. Zepeda, J. Beltrán, M. Maciel, V. Larios y J.Orizaga. "Las Tecnologías de información en pruebas conductuales para el área de neurociencias como impacto social mejorando la calidad de vida". Inventum, vol. 15, no. 28, pp. 76-87. doi: 10.26620/ uniminuto.inventum.15.28.2020.76-87

Editorial: Corporación Universitaria Minuto de Dios - UNIMINUTO.

ISSN: $1909-2520$

eISSN: 2590-8219

Fecha de recibido: 10 de enero del 2020 Fecha de aprobado: 20 de marzo del 2020 Fecha de publicación: 30 de mayo del 2020

Conflicto de intereses: los autores han declarado que no existen intereses en competencia.
Jovan Ricardo Zepeda Gómez ricardozg2491@hotmail.com https://orcid.org/0000-0003-3747-8991 Universidad de Guadalajara, México.

Jesús Raúl Beltrán Ramírez raul.beltran@academicos.udg.mx https: / / orcid.org/0000-0001-8645-9258 Universidad de Guadalajara, México.

Ma. Del Rocío Maciel Arellano ma.maciel@academicos.udg.mx https://orcid.org/0000-0002-5548-2073 Universidad de Guadalajara, México.

Víctor Manuel Larios Rosillo victor.larios@academicos.udg.mx https://orcid.org/0000-0002-2899-724X Universidad de Guadalajara, México.

José Antonio Orizaga Trejo jaorizaga@cucea.udg.mx https://orcid.org/0000-0001-5649-5514 Universidad de Guadalajara, México.

\section{El uso de tecnologías de información en pruebas conductuales para el área de neurociencias como impacto social mejorando la calidad de vida}

\author{
The use of information technology in \\ behavioral tests for neuroscience with \\ social impact to improve life quality
}

\section{O uso da tecnologia da informação em testes comportamentais da neurociência com impacto social para melhorar a qualidade de vida}

\begin{abstract}
Resumen
Las pruebas conductuales en modelos animales permiten estudiar los efectos de distintas sustancias en el sistema nervioso central, probar tratamientos y procedimientos a diversas afecciones relacionadas con el déficit de funciones cognitivas que se presentan, tanto en animales como humanos, y por consecuencia, lograr un mejor entendimiento de estas, por ejemplo, el proceso de memoria, aprendizaje y sus mecanismos básicos.

Se considera una necesidad actual la implementación de la tecnología, ya que otorga mejoras a los procesos de recolección, análisis y procesamiento de datos provenientes del desarrollo de las pruebas, con esto se vuelven más eficientes los ensayos, al reducir el tiempo de estudio al necesario para la ejecución del experimento.

En el presente artículo se expone un breve acercamiento a las pruebas conductuales realizadas en algunos de los modelos animales más utilizados en el área de investigación biomédica y como la tecnología desarrollada en el área proporciona herramientas para la recolección y estudio de la información proveniente de dichas pruebas, mediante el uso de sistemas de visión artificial, análisis computacional de vocalizaciones animales, lectura de señales electrofisiológicas, entre otros.
\end{abstract}

Palabras clave: lectura de señales electrofisiológicas, modelos animales, pruebas conductuales, tecnología, visión artificial. 


\begin{abstract}
Behavioral tests in animal models allow studying the effects of different substances in the central nervous system, test treatments and procedures to various conditions related to the deficit of cognitive functions that occur in both, animals and humans. Consequently, achieve a better understanding of tests, for example, the process of memory, learning and its basic mechanisms.

Being a current need the implementation of technology, provides improvements to the processes of collection, analysis and processing of data from development of tests, making trials more efficient by reducing the study time to the necessary for the execution of the experiment.

In the present work a brief approach is exposed to the behavioral tests carried out in some of the animal models more used in the area of biomedical research and as the technology developed in the area provides tools for the collection and study of the information coming from said tests. through the use of artificial vision systems, computational analysis of animal vocalizations, reading of electrophysiological signals, among others.
\end{abstract}

Keywords: reading of electrophysiological signals, animal models, behavioral tests, technology, artificial vision.

\section{Resumo}

Os testes comportamentais em modelos animais permitem estudar os efeitos de diferentes substâncias no sistema nervoso central, testar tratamentos e procedimentos para diversas condições relacionadas ao déficit de funções cognitivas que ocorrem em animais e humanos. Conseqüentemente, obtenha uma melhor compreensão dos testes, por exemplo, o processo de memória, aprendizado e seus mecanismos básicos.

Sendo uma necessidade atual a implementação de tecnologia, proporciona melhorias nos processos de coleta, análise e processamento de dados a partir do desenvolvimento de testes, tornando os ensaios mais eficientes, reduzindo o tempo de estudo ao necessário para a execução do experimento.

No presente trabalho, uma breve abordagem é exposta aos testes comportamentais realizados em alguns dos modelos animais mais utilizados na área de pesquisa biomédica e, como a tecnologia desenvolvida na área fornece ferramentas para a coleta e o estudo das informações oriundas da referida testes. através do uso de sistemas de visão artificial, análise computacional de vocalizações de animais, leitura de sinais eletrofisiológicos, entre outros.

Palavras - chave: leitura de sinais eletrofisiológicos, modelos animais, testes comportamentais, tecnologia, visão artificial. 


\section{INTRODUCCIÓN}

Las pruebas conductuales constituyen un instrumento importante para el estudio de memoria y aprendizaje, facilitan la comprensión del funcionamiento de estos mecanismos en el sistema nervioso central [1]. En estas es posible observar cambios favorables respecto a habilidades cognitivas o memoria espacial después de algún tratamiento nutricional o médico, lo que mejora significativamente la calidad de vida [2].

Dichas pruebas se categorizan en 2 grupos, modelos animales de respuesta condicionada, aquellas que presentan un estímulo aversivo externo, y no condicionada, donde el estímulo se presenta en la novedad del entorno, generalmente utilizadas en animales [1].

Los modelos animales permiten comprender los trastornos psicológicos en humanos y sus características por la similitud que existe entre el sistema nervioso central de roedores y humanos [3].

Cabe mencionar que la realización de pruebas conductuales en modelos animales, comprende estándares y procesos establecidos que se realizan a mano, los protocolos para su ejecución no comprenden ningún tipo de implementación tecnológica, por lo que presentan periodos prolongados para ejecución, desarrollo, recabado de datos e interpretación de resultados, ejemplo de dichos estándares se observan en la implementación del laberinto abierto de Barnes o el laberinto acuático de Morris, donde los laberintos necesarios para la realización de ensayos, presentan diseños minimalistas sin implementaciones tecnológicas de por medio.

La tecnología presenta alternativas de implementación que permiten la recolección de datos de una manera sencilla en la realización de pruebas mediante utilización de herramientas computacionales de visión artificial como la diferenciación de cuadros en video para seguimiento del animal, identificación de su posición con respecto del entorno, trazado de trayectorias, análisis de audio mediante estudio de amplitud y frecuencia de ondas para estudio de ansiedad, entre otros [4] [5].

Otro factor importante presente en el área de neurociencias es el estudio de señales electrofisiológicas donde el uso de electrodos en el cerebro para lectura electroencefalográfica presenta un ejemplo tecnológico comúnmente utilizado y de elaboración relativamente sencilla, en el caso de animales, es común implementar electrodos profundos mientras que en humanos son superficiales no invasivos [6].

Si bien el desarrollo de recursos tecnológicos en el área ha tenido un impacto importante en los últimos años, en la actualidad existe un déficit de recursos para su implementación, por lo que realizar una comparativa entre desarrollos actuales permitirá futuras mejoras en prototipos funcionales a desarrollar.

A continuación, se expone un breve acercamiento a algunos desarrollos tecnológicos para recolección, análisis y procesamiento de información inspirados en distintos modelos animales en el área de neurociencias con la finalidad de exponer diferentes recursos que permiten volver más eficiente el proceso y facilitar el estudio de memoria, aprendizaje y ansiedad, además de la comprensión de los mecanismos básicos que componen a las funciones cognitivas básicas, gracias a la implementación de tecnologías de información y comunicación, además de exponer las fortalezas y carencias de cada desarrollo, lo que permite abrir un área de oportunidad de desarrollo tecnológico para futuras implementaciones, a fin de cubrir necesidades emergentes en el estudio de modelos animales.

\section{PRUEBAS CONDUCTUALES}

El estudio de memoria y aprendizaje en modelos animales presenta una perspectiva clara del funcionamiento de estos mecanismos y como intervienen en el comportamiento ante la presencia de estímulos externos o la novedad que presenta el entorno [1]. Además, la implementación de dietas específicas en especímenes que se encuentran en la ejecución de los ensayos, generan resultados relevantes en mejora de padecimientos relacionados con memoria espacial y déficit en habilidades cognitivas [2].

Principalmente, existen 2 grupos o vertientes de este estudio que, por la naturaleza que presenta en la ejecución de las pruebas, pueden ser catalogados en modelos de respuesta condicionada y no condicionada [1].

Al hacerse presente el instinto de supervivencia ante la presencia de un peligro potencial, es posible observar el comportamiento animal básico y por tanto estudiar el estado de ansiedad en ambientes controlados, lo que permite a las pruebas conductuales mostrar los efectos a estímulos sobre el cerebro y el sistema nervioso central, en 
general, y permite una mejor comprensión de los trastornos psicológicos en humanos [3].

Por otra parte, cabe destacar que, en el área de neurobiología, son utilizadas para la observación de presencia de neurotransmisores bajo estímulos en ambientes controlados, muestra la relación presente entre la ansiedad y la anatomía de las emociones, causa de los sustratos presentes en la actividad neuronal [7]. Otro trastorno presente es la depresión que, debido al estrés presente en las pruebas de respuesta condicionada vuelve susceptibles a los individuos a padecerla [8].

Cabe señalar que, para el tratamiento de los trastornos neurológicos y enfermedades neurodegenerativas, la realización de pruebas de fármacos emergentes en modelos animales presenta una aproximación a los efectos en seres humanos, por lo que las pruebas de bioequivalencia ofrecen información importante acerca de tratamientos que mejoren la condición en pacientes que padezcan dichos trastornos. Las pruebas de bioequivalencia en conjunto con la ejecución de pruebas conductuales en modelos animales constituyen un importante recurso en la validación de fármacos emergentes, como nuevas soluciones o genéricos de los ya existentes, que garantiza el mismo efecto durante el tratamiento que un medicamento de patente [9].

Según la norma oficial mexicana NOM-177SSA1-2013, publicada en el Diario Oficial de la Federación en 2013 , se establecen una serie de protocolos o estándares a seguir en la prueba de fármacos en estudios de bioequivalencia y biocompatibilidad que se deben cumplir en aquellos modelos animales utilizados para estudios preclínicos con posibilidad de migrar a pruebas en seres humanos [10].

\section{A. Relación entre los modelos animales y humanos}

Los modelos animales permiten mejorar la comprensión, causa, diagnóstico y tratamiento a distintos padecimientos dentro de investigación biomédica, lo que abre el panorama a diferentes soluciones ante la presencia de afecciones tanto de animales como de humanos, y ofrece aportes relevantes en áreas docentes y disciplinas científicas, en las cuales, hasta la fecha es indispensable la implementación de animales [11].

Al ser un reactivo biológico, es indispensable garantizar la pureza o estandarización en la producción de los animales, que son criados en ambientes controlados y presenten condiciones biológicas y sanitarias estandarizadas [11].

Como objetivo principal de utilizar modelos animales dentro del proceso de investigación es el de comprender los efectos de la implementación de sustancias o procedimientos sobre un sistema biológico similar al del ser humano [12].

\section{B. Ejemplos de antecedentes generales e implementación tecnológica}

\section{Laberinto abierto de Barnes}

Dentro de los modelos animales de respuesta no condicionada más utilizados para realización de pruebas conductuales, se destaca el laberinto abierto de Barnes, que por su configuración permite el estudio de la memoria espacial. Consta principalmente de una superficie circular elevada con orificios circulares en su periferia, según sus variantes más utilizadas en la realización de ensayos, puede contener desde 8 a 32 orificios [13].

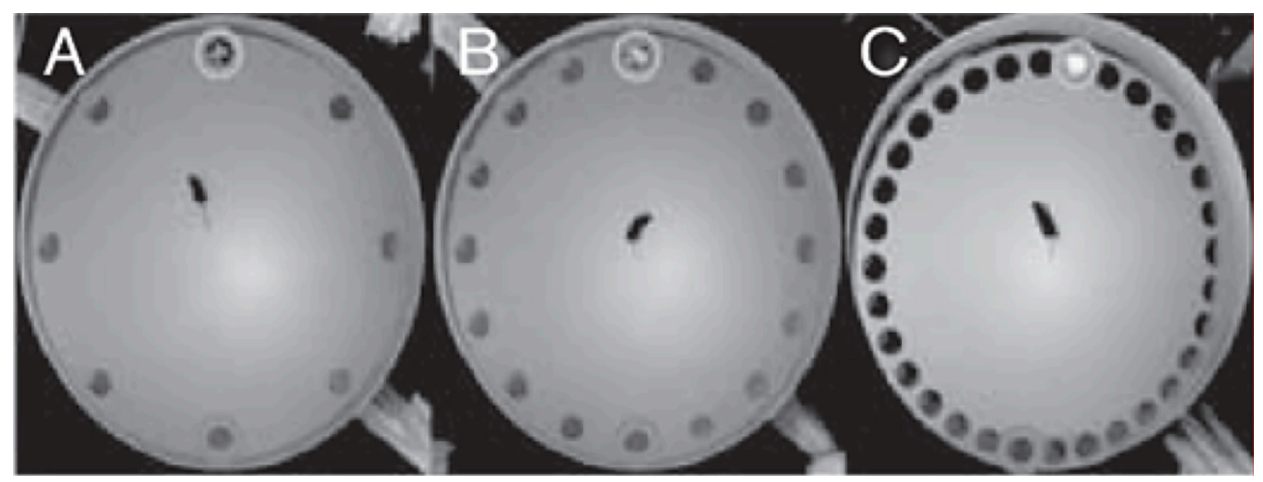

Figura 1. Variantes sobre laberinto abierto de Barnes.

Fuente: [13]. 
Un ejemplo de software para el registro de datos sobre esta plataforma es el caso de "TSE VideoMot 2", que se expone en la siguiente figura, el cual cuenta con funciones de calibración para dimensiones de la plataforma, regiones de interés como el caso de los orificios y seguimiento del animal [14]. Otro programa similar, desarrollado en Java por [15], presenta una alternativa tecnológica para la realización de ensayos sobre el laberinto abierto de Barnes [15].

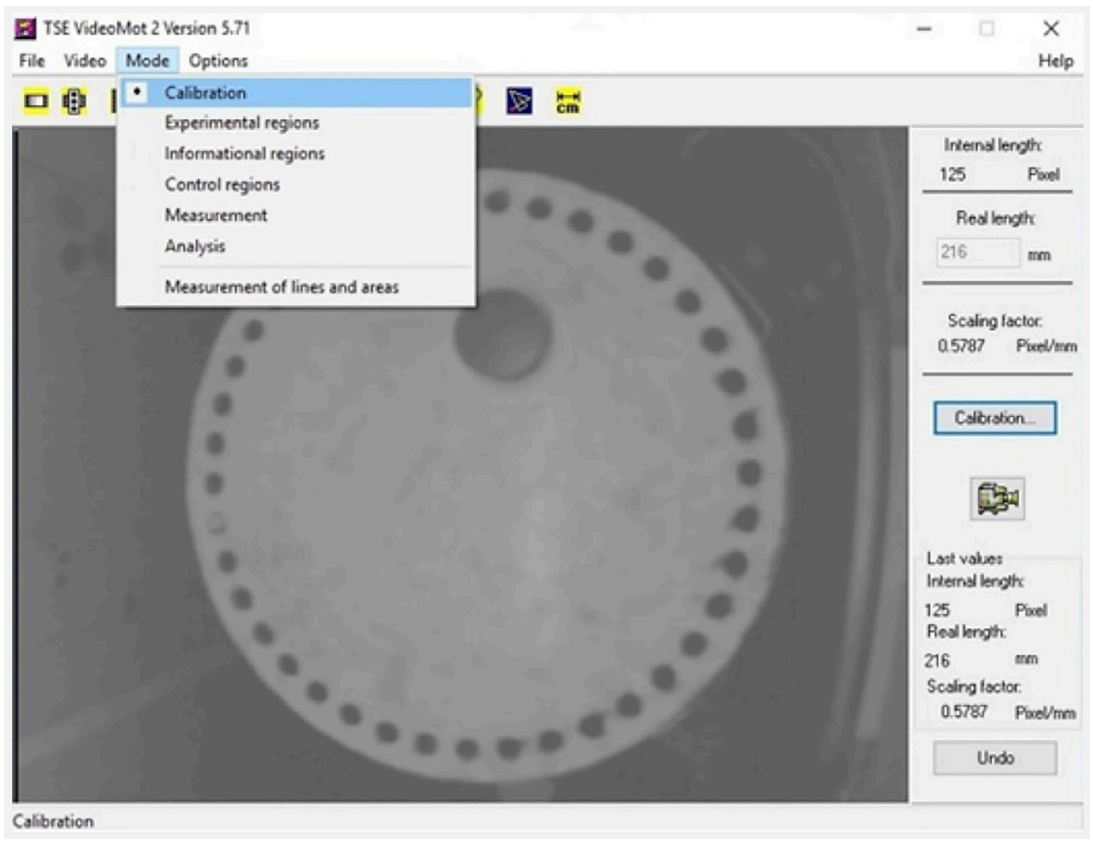

Figura 2. Software TSE VideoMot 2.

Fuente: [14].

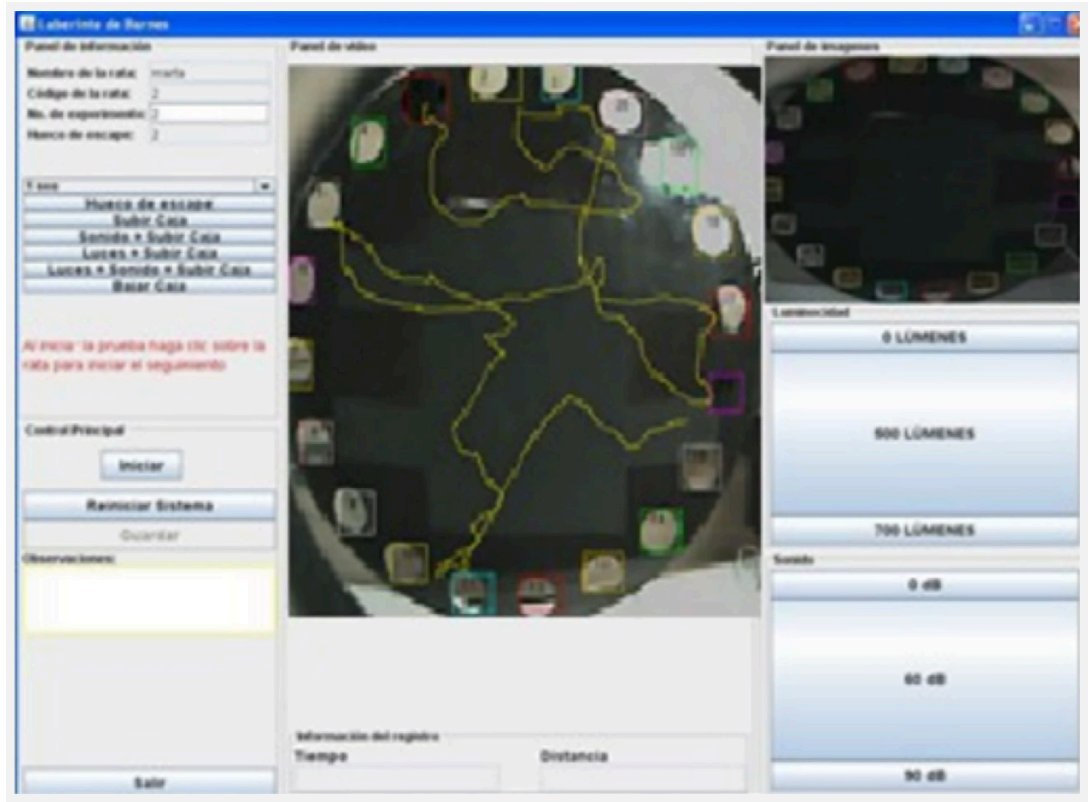

Figura 3. Ejemplo de software realizado en Java para seguimiento en laberinto Abierto de Barnes.

Fuente: [15]. 


\section{Laberinto acuático de Morris}

El laberinto acuático de Morris frecuentemente es utilizado para analizar y evaluar las funciones cognitivas del animal y su capacidad en cuestión a memoria y aprendizaje [5].

Básicamente se le puede describir como un contenedor de color oscuro con base circular, dividido en cuadrantes, lleno de líquido en tres cuartas partes aproximadamente de su altura total, cuenta con una pequeña base situada en alguno de los cuadrantes, apenas visible para el roedor, brindándole pequeños periodos de descanso en su recorrido [5].
Una de las principales características a registrar en video en la ejecución de la prueba es el recorrido que realiza el animal durante la búsqueda de la base. Mediante el uso de software especializado en seguimiento de objetos, con la utilización del principio de diferenciación de cuadros, permite seguir al animal mediante la resta de características similares entre el cuadro anterior y actual del video [5].

En la siguiente figura se aprecia el diagrama de funcionamiento del software para diferenciación de cuadros y el trazado de la trayectoria registrada por parte del programa.

(a)

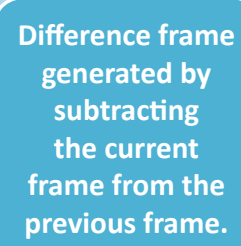

Remove

objects in a

binary image

that are too

small.
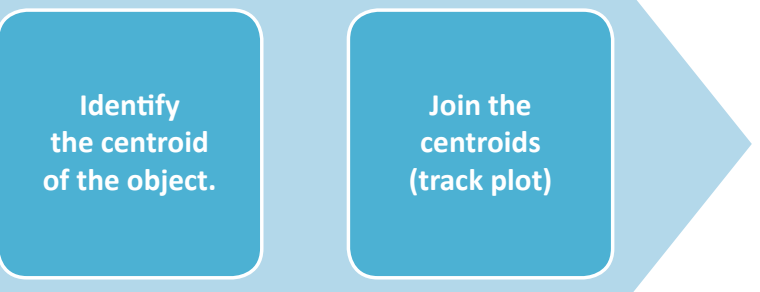

(b)

(i)
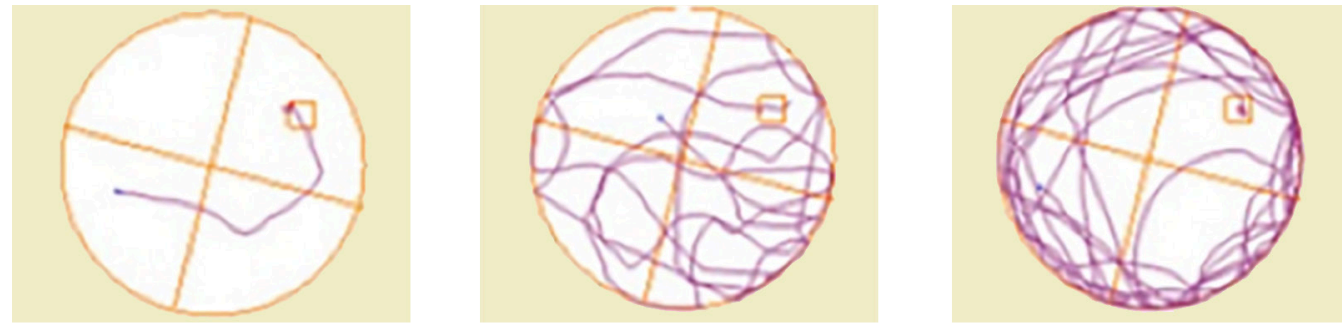

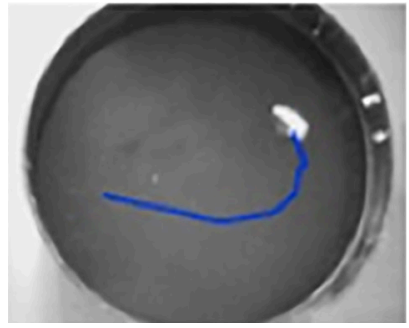

Group 1: Control

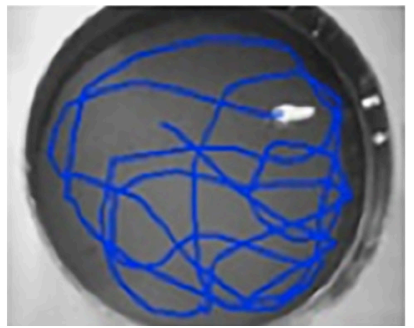

Group 2: PTZ

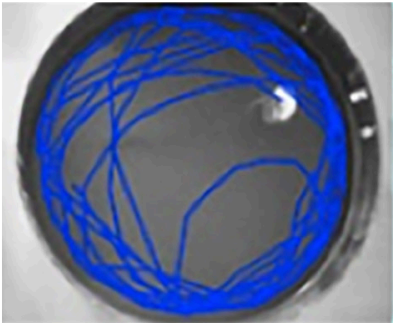

Group 3: Scopolamine

Figura 4. Diagrama de funcionamiento del software de diferenciación de cuadros (a) y trazado de la trayectoria sobre el seguimiento del animal (b). Fuente: [5]. 


\section{Cámara de condicionamiento operante}

Por su parte, la cámara de acondicionamiento operante de Skinner es considerada como uno de los modelos más importantes de respuesta emocional condicionada, basándose en el comportamiento asociativo al contar con la naturaleza pavloviana de estímulo-respuesta como principio fundamental en la realización de esta prueba.
Al contar con una palanca accionada por el animal, permite premiarlo con alimento o castigarlo con la implementación de sonidos agudos, aire a presión o una corriente eléctrica [1].

La tecnología toma importancia en el desarrollo de los ensayos en este modelo al ser implementadas técnicas de visión artificial, un ejemplo de lo anterior es un sistema de seguimiento en video, mostrado en la siguiente figura [4].

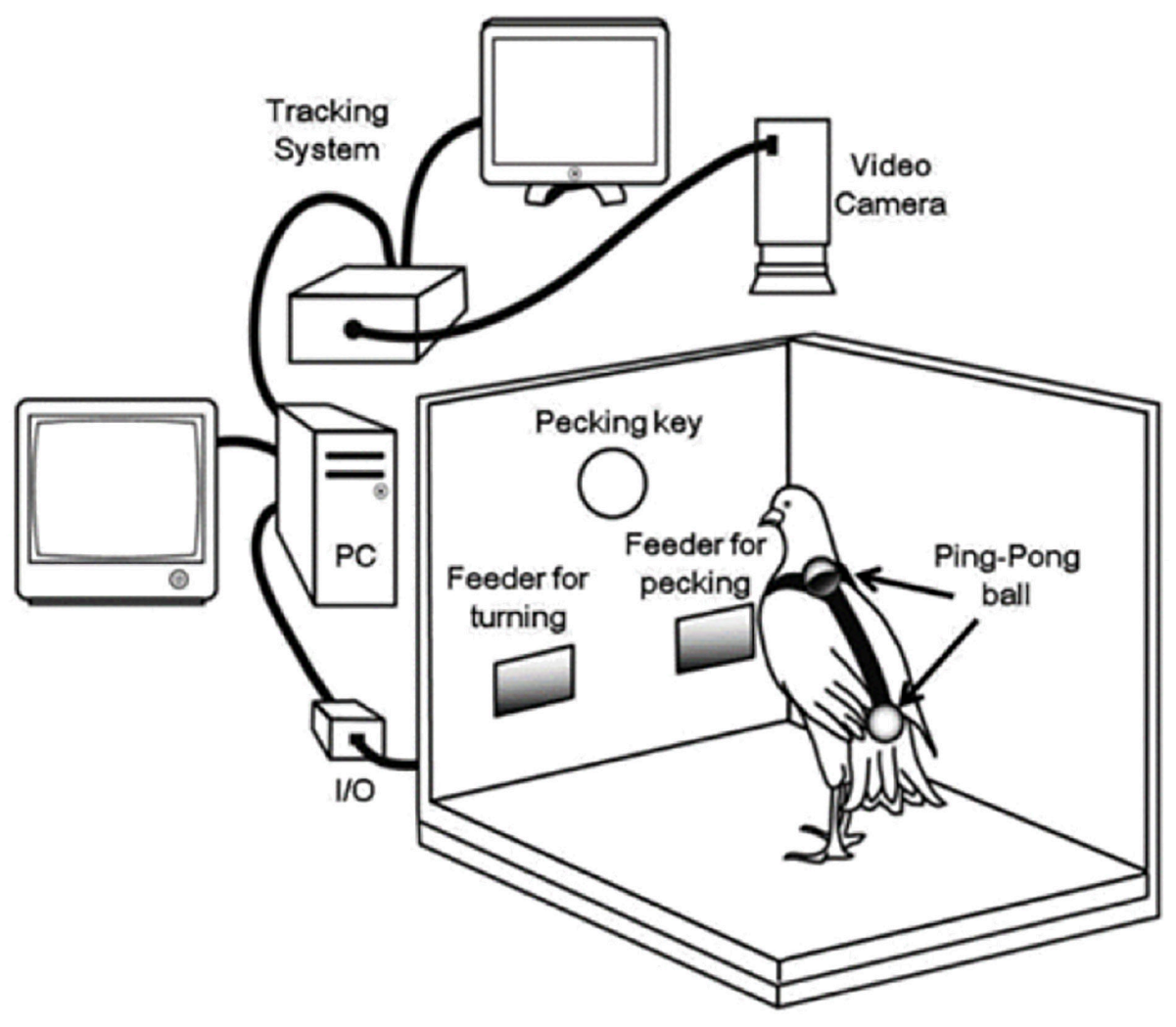

Figura 5. Diagrama de operación Sistema de un sistema de seguimiento.

Fuente: [4].

El sistema permite reconocer 2 pelotas de pingpong colocadas estratégicamente en el animal, con la finalidad de utilizarlos como puntos auxiliares o marcas para identificar el centroide del espécimen, la información recabada sobre la posición de las pelotas es comunicada a un ordenador para su procesamiento, y de esa manera, identificar su orientación dentro de la caja de Skinner [4].

La vocalización animal ofrece información relevante sobre el estudio de ansiedad en la realización de pruebas conductuales, por lo que es importante el registro de la respuesta vocal del animal. Mediante el procesamiento computacional de frecuencia, tiempo y amplitud de onda procedente del sonido registrado, es posible identificar la respuesta sensitiva ante contingencias operantes en el desarrollo del experimento [4].

Manabe expone una adaptación al sistema anterior para el registro de vocalización animal y su estudio basado en el análisis de señales digitales mediante transformada rápida de Fourier, mostrada en la siguiente ilustración [4]. 


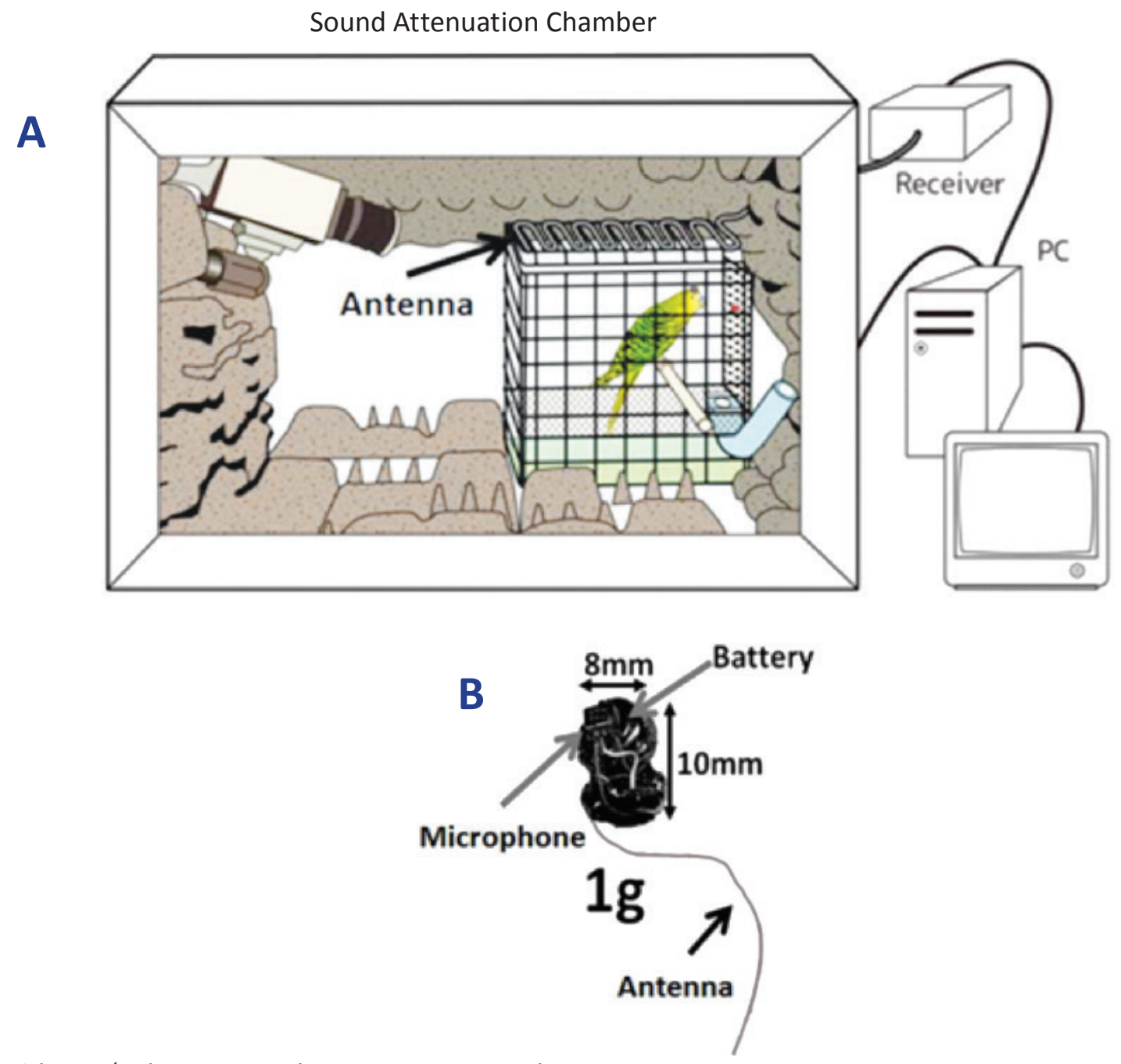

Figura 6. Adaptación de un sistema de reconocimiento vocal. Fuente: [4].

\section{DESARROLLOS REALIZADOS PARA IMPLEMENTACIÓN EN DIFERENTES PLATAFORMAS PARA ESTUDIO DE DISTINTOS ORGANISMOS}

A medida que los procesos y métodos de inteligencia artificial se hacen presentes en los modelos de pruebas conductuales es posible desarrollar sistemas que permitan realizar tareas más complejas como es el caso del ejemplo que presenta Geuther en 2019, sobre un sistema de seguimiento robusto en ambientes complejos, donde, por medio de la implementación de redes neuronales y con un mínimo entrenamiento por parte del sistema es posible obtener resultados más tangibles en un menor tiempo durante la realización de pruebas conductuales [16].
Otro ejemplo que cabe mencionar dentro de las tecnologías aplicadas al análisis del comportamiento es el propuesto por Egnor y Branson, donde, mediante técnicas de ciencias computacionales como el procesamiento de imágenes, logran estudiar el comportamiento o conducta de los animales [17].

Pensando en que la tecnología es adaptable y presenta una versatilidad considerable para su implementación en distintos escenarios sin presentar cambios fuertes, abre un panorama más amplio, en el cual no solo es posible implementar tecnología para pruebas clásicas, como las presentadas en puntos anteriores, sino experimentar sobre distintos organismos como peces, insectos, anfibios o especies pequeñas en general, con un mismo sistema [18], [19]. 

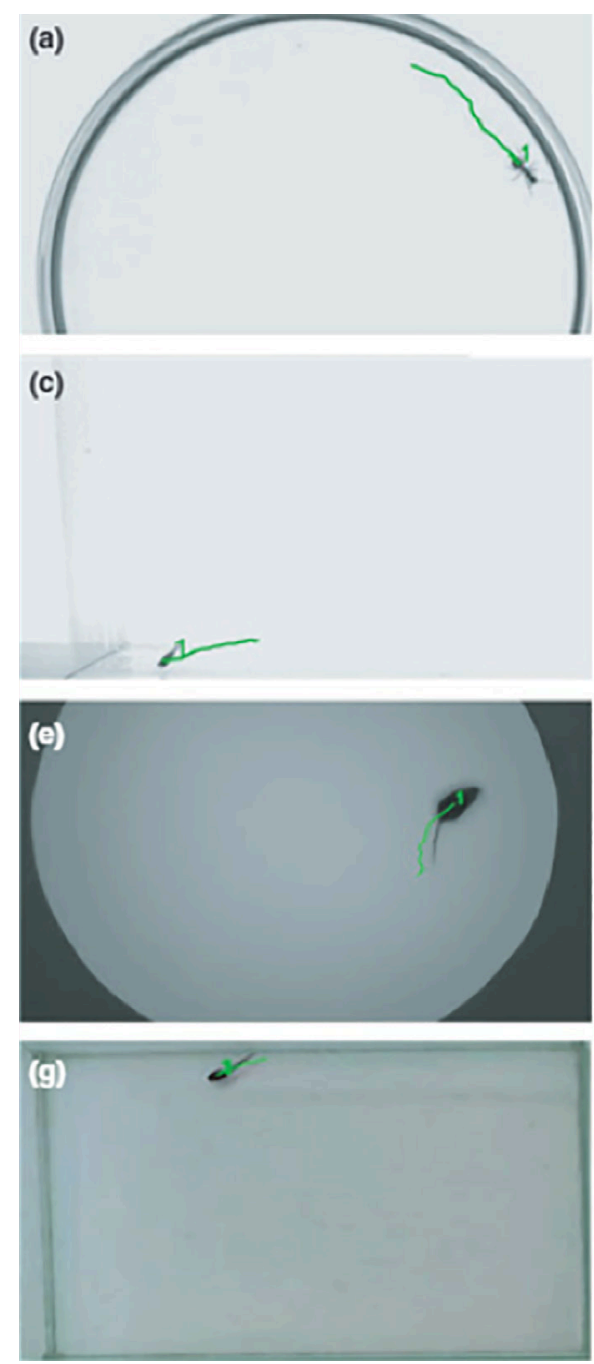

Figura 7. Seguimiento de la trayectoria realizada por distintos organismos. Donde:

a) hormiga, b) cucaracha, c) pez guppy, d) dos peces guppy, e) ratón, f) salmon, g) renacuajo y d) pez cebra.

Fuente: [19].
Un recurso importante dentro del área de neurociencias para la comprensión de la estructura del conocimiento y el comportamiento es el estudio de la conducta en roedores, donde el lenguaje corporal juega un papel importante, permite entender los procesos implicados en un análisis conductual, desde las posturas que toma el animal hasta el catálogo completo de patrones de acción que pudiera presentar el roedor. Al incluir recursos computacionales como máquinas de aprendizaje y usar paradigmas como el aprendizaje supervisado y no supervisado, donde el principal método de entrenamiento de la máquina de aprendizaje es la creación de relaciones de preguntas y respuestas en el caso del aprendizaje supervisado, ofrece una herramienta de predicción sobre la presencia
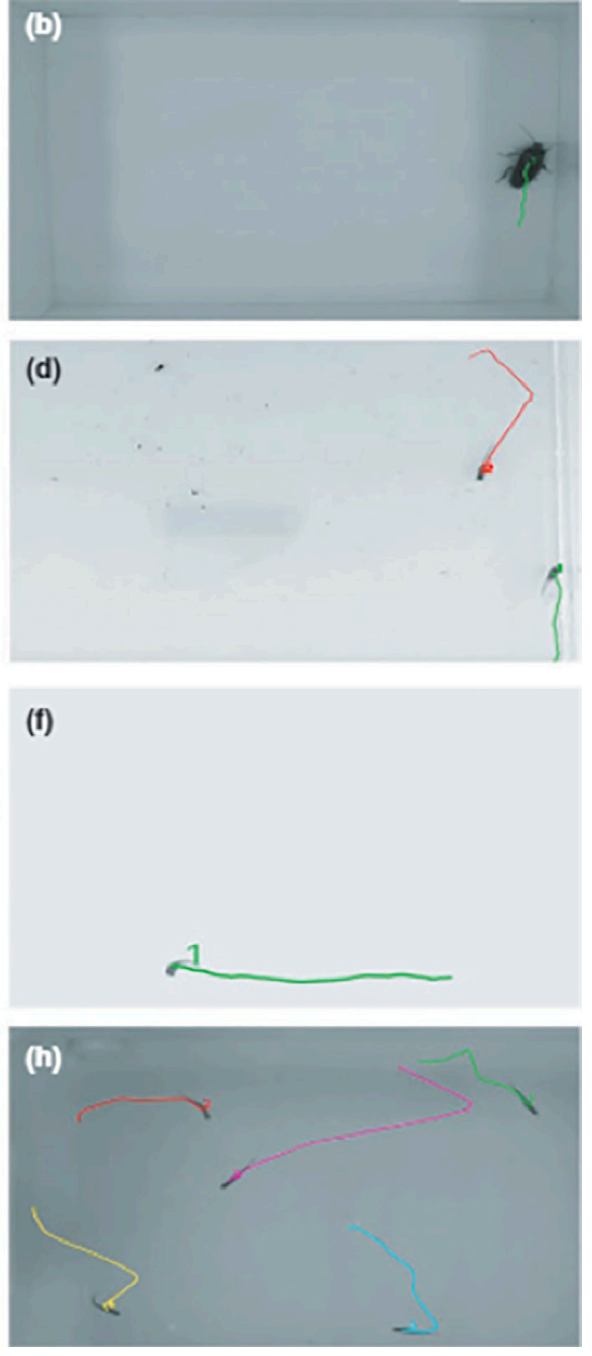

(f)

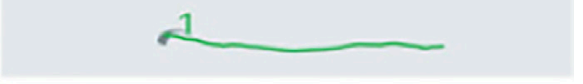


la implementación, tanto del sistema desarrollado, como del tratamiento implementado durante la prueba [6].

En el área de neurociencias, la tecnología permite la versatilidad en la implementación de pruebas conductuales en distintos modelos animales, lo que permite estudiar varias variables con el mismo equipo, que va desde el uso de sistemas de visión artificial para seguimiento de trayectorias, hasta el uso de electrodos profundos para el estudio de señales electroencefalográficas para medir la actividad eléctrica en el cerebro, producida por estímulos externos [6].

Entre las principales ventajas de la implementación tecnológica, cabe destacar el proceso automatizado para la recolección y procesamiento de la información recabada, que hace innecesaria la intervención de un observador experto [6].

\section{CONCLUSIONES}

Las pruebas conductuales constituyen una herramienta importante para la comprensión de las funciones cognitivas y sus características presentes tanto en animales como en humanos, además de facilitar el estudio de procedimientos o tratamientos y de los efectos que se presentan en organismos similares. Al implementar tecnología sobre ellas, permite facilitar la adquisición de información, vuelve más eficiente el proceso y consecuentemente facilita el estudio de memoria y aprendizaje sobre ambientes controlados.

Con el continuo desarrollo de fármacos emergentes, es de vital importancia validar su eficacia en el tratamiento de enfermedades neurodegenerativas mediante la implementación de pruebas conductuales en modelos animales, lo que permite el estudio y respuesta del sistema nervioso central durante el desarrollo de las pruebas y la aplicación del fármaco. Las implementaciones y desarrollos tecnológicos sobre las pruebas conductuales en condiciones controladas facilitan de manera considerable la obtención de resultados fidedignos en tiempos reducidos.

Debido a que es un proceso que se realiza de manera artesanal, la implementación de pruebas conductuales en modelos animales presenta ciertas deficiencias, tanto en la ejecución como en la recolección de datos, lo cual se traduce en periodos prolongados de estudio, que pueden ser de semanas o incluso meses, por lo que la implementación de tecnologías emergentes se convierte en una necesidad al mejorar la eficiencia de dicho proceso.

A pesar que la tecnología se hace presente con mayor frecuencia en distintas áreas de estudio, al presentar enfoques multidisciplinarios y cubrir las necesidades presentes en este tipo de pruebas, actualmente, los desarrollos tecnológicos siguen siendo escasos y aún presentan ciertos inconvenientes para dar un correcto seguimiento durante la realización de pruebas.

Existen diversas técnicas del área de ciencias computacionales que aseguran la integridad de los datos a colectar, entre los cuales se destaca el uso e implementación de técnicas de visión artificial y procesamiento de imágenes, estudio de frecuencias y longitud de onda a partir de vocalizaciones en animales, entre otros.

Cabe destacar que la recolección, análisis y procesamiento de los datos recabados mediante dispositivos tecnológicos, permite reducir tiempos de ejecución y costos, además facilita el desarrollo de las pruebas y permite eliminar el factor de error humano, para obtener resultados tangibles y volver el proceso más eficiente.

Tomando como referente el procesamiento continuo de imágenes como una de las herramientas computacionales más utilizadas en plataformas destinadas al estudio del comportamiento, cabe destacar la versatilidad que presenta en el estudio de diversos organismos, lo que permite ampliar el panorama, ofrece un recurso importante para estudios clásicos del comportamiento como es el caso del laberinto abierto de Barnes, laberinto acuático de Morris, entre otros. También es un instrumento de recolección de información, de nuevos estudios y pruebas emergentes en los últimos años.

Aunque el estudio de las vocalizaciones y el comportamiento ante distintos estímulos en pruebas conductuales no es un caso reciente, la implementación de equipos tecnológicos permite recabar información procedente de varias variables al mismo tiempo y procesarla a fin de lograr una mayor comprensión de los mecanismos que intervienen en la respuesta que presenta cada organismo ante la situación presente en cada ensayo realizado.

Al realizar un estudio sobre las tecnologías existentes para la recolección, análisis y procesamiento de datos en pruebas conductuales, es posible 
ofrecer un acercamiento de las herramientas computacionales destinadas al apoyo del área de neurociencias y, por tanto, darles difusión entre los principales centros de investigación con la finalidad de abrir la posibilidad de su implementación. A su vez, permite abrir el panorama general, facilita reconocer las carencias de desarrollos existentes, y ofrece la posibilidad de innovar en futuros prototipos o software especializado.

\section{REFERENCIAS}

[1] L. A. Polanco, I. C. Vargas y M. E. Góngora. (2011). "Modelos animales: una revisión desde tres pruebas utilizadas en ansiedad", Suma Psicológica. Vol. 18, $\mathrm{n}^{\circ}$ 2, pp. 141-148. [En línea]. Disponible en: http://www.scielo.org. co/pdf/sumps/v18n2/v18n2a11.pdf

[2] L. O. Aguirre López, J. L. Chávez Servia, C. C. Gómez Rodiles, J. R. Beltrán Ramírez y J. Bañuelos Pineda. (2017). "Blue Corn Tortillas: Effects on Learning and Spatial Memory in Rats", Plant Foods for Human Nutrition. Vol. 72, n 4, pp. 448-450. [En línea]. Disponible en: https: / /www. researchgate.net/publication/ 321754226_Blue_corn_tortillas_Effects_ on_learning_and_spatial_memory_in_rats. doi:https://doi.org/10.1007/S11130-01 7-0642-1

[3] A. Mora-Gallegos, y S. Salas Castillo. (2014). "Modelos animales de miedo y ansiedad: descripciones neuro-conductuales", Actualidades en Psicología. Vol 28, $\mathrm{n}^{\circ}$ 117, pp. 1-12. [En línea]. Disponible en: https://revistas. ucr.ac.cr/index.php/actualidades/article/ view/14595. doi:http://dx.doi.org/10.15517 /ap.v28i117.14595

[4] K. Manabe. (2017). "La evolución de la caja de skinner para detectar movimiento y vocalización", Revista Mexicana de Análisis de la Conducta. Vol. 43, $\mathrm{n}^{\circ}$ 2, pp. 192-211. [En línea]. Disponible en: http://www.revistas.unam. $\mathrm{mx} /$ index. $\mathrm{php} / \mathrm{rmac} /$ article / view/62313/55040. doi:https://doi.org/10. 5514/rmac.v43.i2.62313

[5] S. Singh, H. Kaur, y R. Sandhir. (2016). "Fractal dimensions: A new paradigm to assess spatial memory and learning using Morris water maze", Behavioural Brain Research.
N²99, pp. 141-146. [En línea]. Disponible en: http://europepmc.org/article/med/265 92165. doi:https://doi.org/10.1016/j.bbr.20 15.11 .023

[6] P. Vieyra Reyes, C. Jiménez Garcés, M. M. Hernández González y A. Hernández Alva. (2012). "Bioingeniería aplicada a modelos conductuales en animales de experimentación", Ideas en Ciencia. pp. 46-54. [En línea]. Disponible en: http://ri.uaemex.mx/handle /20.500.11799/49947

[7] B. Cedillo Ildefonso. (2017). "Generalidades de la neurobiología de la ansiedad", Revista Electrónica de Psicología Iztacala. Vol. 20, $\mathrm{n}^{\circ}$ 1, pp. 239-251. [En línea]. Disponible en: http://www.medigraphic.com/pdfs/epsico logia/epi-2017/epi171m.pdf

[8] A. Sequeira Cordero y J. Fornaguera Trías. (2014). "Diferencias individuales en modelos animales: un enfoque para el estudio de factores neurobiológicos relacionados con depresión", Actualidades en Psicología: Neurociencia y Psicología. Vol. 28, n 117, pp. 53-66. [En línea]. Disponible en: https://www.researchgate. net/publication/277234076_Diferencias_ individuales_en_modelos_animales_un_ enfoque_para_el_estudio_de_factores_ neurobiologicos_relacionados_con_depresion_ Individual_differences_in_animal_models_an approach_to_study_neurobiologi. doi:http:// dx.doi.org/10.15517/ap.v28i117.14115

[9] F. E. Estévez Carrizo. (2000). Estudios de bioequivalencia: enfoque metodológico y aplicaciones prácticas en la evaluación de medicamentos genéricos. Rev Med Uruguay, 16(2), 133-143. [En línea]. Disponible en: http: / / www.rmu.org.uy/revista/2000v2/ art5.pdf

[10] DOF. Norma Oficial Mexicana NOM-177SSA1-2013. Ciudad de México: Diario Oficial de la Federación, 2013. [En línea]. Disponible en: http://www.dof.gob.mx/nota_detalle. php?codigo $=5314833 \&$ fecha $=20 / 09 / 2013$

[11] S. Hernández. (2006). “El modelo animal en las investigaciones biomédicas", Biomedicina. Vol. 2, $\mathrm{n}^{\circ}$ 3, pp. 252-256. [En línea]. Disponible en: http://www.um.edu.uy/docs/revistabiomedicina/2-3/A\%F102\%20N\%BA3\%20 Dic2006. pdf\#page $=52$ 
[12] V. D. Garzón Cortés, “Modelos Utilizados en Investigación Biomédica", en Seminario de Biotecnología y Bioseguridad de OGM's. [En línea]. Disponible en: https://www. conacyt.gob.mx/cibiogem/images/cibio gem/Herramientas-ensenanza-investigacion/ Seminarios/Docs/biomedicina-seminario 250915.pdf

[13] G. Riedel, L. Robinson \& B. Crouch. (2018). "Spatial learning and flexibility in 129S2/ SvHsd and C57BL/6J mouse strains using different variants of the Barnes maze", Behavioural Pharmacology. Vol. 29, $\mathrm{n}^{\circ}$ 8, pp. 688-700. [En línea]. Disponible en: https:// abdn.pure.elsevier.com/en/publications/ spatial-learning-and-flexibility-in129s2svhsd-and-c57bl6j-mouse-. doi:https:// doi.org/10.1097/FBP.0000000000000433

[14] M. W. Pitts (2018). "Barnes Maze Procedure for Spatial Learning and Memory in Mice", Bioprotocol. Vol. 8, $\mathrm{n}^{\circ}$ 5. [En línea]. Disponibleen: https: / / bio-protocol.org/e2744.doi:10. 21769/BioProtoc.2744

[15] C. A. Puentes Morales. (2017). "Sistema de control para mapeo, estímulos lumínicos y sonoros en el laberinto circular de Barnes", Visión Investigadora. $\mathrm{N}^{\circ}$ 1. [En línea]. Disponible en: https://dialnet.unirioja.es/ servlet/articulo?codigo $=6080425$

[16] B. Q. Geuther, S. P. Deats, , Fox, K. J., Murray, S. A., Braun, R. E., White, J. K., V. Kumar. (2019). "Robust mouse tracking in complex environments using neural networks",
Communications Biology volume. Vol. 2, $\mathrm{n}^{\circ}$ 124, pp. 1-11. [En línea]. Disponible en: https: / / www.nature.com/articles/s42003019-0362-1. doi:https://doi.org/10.1038/ s42003-019-0362-1

[17] S.E.Egnor, \& K. Branson. (2016). “Computational analysis of behavior. Annu”, Rev. Neurosci. Vol. 39, pp. 217-236. [En línea]. Disponible en: https: / /www.annualreviews.org/doi/ abs/10.1146/annurev-neuro-070815-013845. doi:10.1146/annurev-neuro-070815-013845

[18] A. Rodríguez, M. Bermúdez, J. Rabuñal, \& J. Puertas, "Fish tracking in vertical slot fishways using computer vision techniques", Journal of Hydroinformatics, vol. 17, pp. 275-292, 2015.

[19] A. Rodriguez, H. Zhang, J. Klaminder, T. Brodin, P. L. Andersson \& M. Andersson. (2017). "ToxTrac: A fast and robust software for tracking organisms", Methods in Ecology and Evolution. Vol. 9, $\mathrm{n}^{\circ}$ 3, pp. 460-464. [En línea]. Disponible en: https: / / besjournals. onlinelibrary.wiley.com/doi/pdf/10.1111/ 2041-210X.12874. doi: https://doi.org/10.11 $11 / 2041-210 X .12874$

[20] K. V. Gris, J. P. Coutu \& G. Denis. (2017). "Supervised and Unsupervised Learning Technology in the Study of Rodent Behavior", Frontiers in Behavioral Neuroscience. Vol. 11, pp. 1-6. [En línea]. Disponible en: https:// www. frontiersin.org/articles/10.3389/ fnbeh.2017.00141/full. doi:10.3389/fnbeh.20 17.00141 